\title{
Proteasome Inhibitors Bortezomib and Carfilzomib Stimulate the Transport Activity of Human Organic Anion Transporter $1^{\mathrm{S}}$
}

\author{
Yunzhou Fan and Guofeng You \\ Department of Pharmaceutics, Rutgers, the State University of New Jersey, Piscataway, New Jersey \\ Received October 22, 2019; accepted March 10, 2020
}

\section{ABSTRACT}

Organic anion transporter 1 (OAT1), expressed at the basolateral membrane of renal proximal tubule epithelial cells, mediates the renal excretion of many clinically important drugs. Previous study in our laboratory demonstrated that ubiquitin conjugation to OAT1 leads to OAT1 internalization from the cell surface and subsequent degradation. The current study showed that the ubiquitinated OAT1 accumulated in the presence of the proteasomal inhibitors MG132 and ALLN rather than the lysosomal inhibitors leupeptin and pepstatin A, suggesting that ubiquitinated OAT1 degrades through proteasomes. Anticancer drugs bortezomib and carfilzomib target the ubiquitin-proteasome pathway. We therefore investigate the roles of bortezomib and carfilzomib in reversing the ubiquitination-induced downregulation of OAT1 expression and transport activity. We showed that bortezomib and carfilzomib extremely increased the ubiquitinated OAT1, which correlated well with an enhanced OAT1-mediated transport of $\mathrm{p}$-aminohippuric acid and an enhanced OAT1 surface expression. The augmented OAT1 expression and transport activity after the treatment with bortezomib and carfilzomib

\section{Introduction}

Many clinically important drugs, including antiviral therapeutics (e.g., adefovir, acyclovir), antitumor drugs (e.g., methotrexate), antibiotics (e.g., cephaloridine, penicillin G), antihypertensives (e.g., captopril, quinapril), and anti-inflammatories (e.g., salicylate, indomethacin), are eliminated from the kidney through organic anion transporter 1 (OAT1) (You, 2004; Burckhardt, 2012; Liang et al., 2015). OAT1, expressed at the basolateral membrane of the renal proximal tubules, actively transports drugs from blood into tubule cells, followed by the efflux of these drugs to urine by other transporters in the apical membrane (You, 2002; Nigam, 2015; Xu et al., 2016a). The function of OAT1 is the rate-limiting factor in the renal clearance of drugs from the body, affecting drug concentration in the plasma and various tissues, thereby influencing drug therapeutic efficacy and the toxicity.

This work was supported by grants (to G.Y.) from National Institute of General Medical Sciences (R01-GM079123 and R01-GM127788).

https://doi.org/10.1124/mol.119.118653.

S This article has supplemental material available at molpharm. aspetjournals.org. resulted from a reduced rate of OAT1 degradation. Consistent with this, we found decreased $20 \mathrm{~S}$ proteasomal activity in cells that were exposed to bortezomib and carfilzomib. In conclusion, this study identified the pathway in which ubiquitinated OAT1 degrades and unveiled a novel role of anticancer drugs bortezomib and carfilzomib in their regulation of OAT1 expression and transport activity.

\section{SIGNIFICANCE STATEMENT}

Bortezomib and carfilzomib are two Food and Drug Administration-approved anticancer drugs, and proteasome is the drug target. In this study, we unveiled a new role of bortezomib and carfilzomib in enhancing OAT1 expression and transport activity by preventing the degradation of ubiquitinated OAT1 in proteasomes. This finding provides a new strategy in regulating OAT1 function that can be used to accelerate the clearance of drugs, metabolites, or toxins and reverse the decreased expression under disease conditions.

ABBREVIATIONS: Cl, confidence interval; DMEM, Dulbecco's modified Eagle's medium; GAPDH, human embryonic kidney 293; HEK293, glyceraldehyde-3-phosphate dehydrogenase; hOAT1, human OAT1; IB, immunoblotting; LDH, lactate dehydrogenase; OAT, organic anion transporter; PAH, p-aminohippuric acid; PBS/CM, PBS/Ca ${ }^{2+/} \mathrm{Mg}^{2+}$; sulfo-NHS-SS-biotin, sulfosuccinimidyl-2-[biotinamido]ethyl-1,3-dithiopropionate (sulfo-NHS-SS-biotin); Ub, ubiquitin; ZIP14, Zrt/IRT-like protein 14. 
that activation of protein kinase C inhibits OAT1 transport activity and reduces the amount of OAT1 at the cell surface by enhancing OAT1 ubiquitination, resulting in accelerated OAT1 internalization from cell surface to intracellular early endosomes and subsequent proteolytic degradation (Zhang et al., 2008, 2013; Xu et al., 2017). Ubiquitination is an important posttranslational mechanism of OAT1 regulation (Xu et al., 2016a; $\mathrm{Xu}$ and You, 2017).

The proteasomes and the lysosomes are two major systems through which cells degrade proteins (Clague and Urbe, 2010). The two proteolytic systems can be differentiated by the sensitivity to corresponding inhibitors. Degradation of polypeptides through the proteasomes can be prevented by proteasomal inhibitors, such as MG132, whereas lysosomal proteolysis can be hindered by lysosomal inhibitors, such as leupeptin (Kisselev and Goldberg, 2001; Goldberg and Rock, 2002; Lee et al., 2011; Yang et al., 2013). Since ubiquitination leads to OAT internalization and degradation, the alteration of proteasome or lysosome activity can potentially affect the transporter function. In the present study, we investigated the proteolytic system in which the ubiquitinated OAT1 is degraded and explored the strategies of reversing ubiquitination-dependent OAT1 degradation and its influence on OAT1 expression and transport activity.

\section{Materials and Methods}

Materials. Human embryonic kidney 293 (HEK293) cells were purchased from American Type Culture Collection (Manassas, VA). ${ }^{3} \mathrm{H}$-labeled p-aminohippuric acid (PAH) was purchased from PerkinElmer (Waltham, MA). Sulfosuccinimidyl-2-[biotinamido]ethyl-1,3dithiopropionate (Sulfo-NHS-SS-biotin), streptavidin-agarose resin, and protein G-agarose were purchased from Thermo Scientific (Rockford, IL). Mouse anti-Myc antibody (9E10) was purchased from Roche (Indianapolis, IN). Mouse anti-E-Cadherin antibody was purchased from Abcam (Cambridge, MA). Mouse anti-ubiquitin antibody, mouse anti-glyceraldehyde-3-phosphate dehydrogenase (GAPDH) antibody, and normal mouse IgG were purchased from Santa Cruz Biotechnology (Dallas, TX). Lactate dehydrogenase (LDH) cytotoxicity assay kit and 20S proteasome assay kit were purchased from Cayman Chemical (Ann Arbor, MI). Bortezomib and carfilzomib were purchased from Cell Signaling (Danvers, MA). MG132, ALLN, leupeptin, pepstatin A, probenecid, and all other reagents were purchased from SigmaAldrich (St. Louis, MO).

Cell Culture. Parental HEK293 cells were cultured in Dulbecco's modified Eagle's medium (DMEM) (Corning, Tewksbury, MA) supplemented with $10 \% \mathrm{FBS}$ (Gibco, Grand Island, NY) at $37^{\circ} \mathrm{C}$ in $5 \% \mathrm{CO}_{2}$. HEK293 cells stably expressing human OAT1 (hOAT1) were generated in our laboratory (Xu et al., 2016b). hOAT1 cells were maintained in DMEM medium supplemented with $0.2 \mathrm{mg} / \mathrm{ml}$ of G418 (Gibco) and $10 \%$ FBS.

Transport Measurement. The transport activity was measured using the method published in our laboratory (Zhang et al., 2008). The uptake solution consisted of $\mathrm{PBS} / \mathrm{Ca}^{2+/} \mathrm{Mg}^{2+}(\mathrm{PBS} / \mathrm{CM})$, and $\left[{ }^{3} \mathrm{H}\right] \mathrm{PAH}$ $(20 \mu \mathrm{M})$ was added to each well. After 3 minutes, the uptake process was stopped by aspirating the uptake solution and rapidly washing the cells with ice-cold PBS solution. The cells were then solubilized in $0.2 \mathrm{~N} \mathrm{NaOH}$, neutralized in $0.2 \mathrm{~N} \mathrm{HCl}$, and aliquoted for liquid scintillation counting (LS650; Beckman).

Lactate Dehydrogenase Cytotoxicity Assay. Cytotoxicity of bortezomib and carfilzomib was assayed using LDH cytotoxicity assay kit (Cayman Chemical) according to the manufacturer's protocol. After treatment with bortezomib or carfilzomib for 12 hours, $100 \mu \mathrm{l}$ of supernatant of hOAT1 cells was transferred to a 96 -well assay plate, and $100 \mu \mathrm{l}$ of LDH reaction solution was added and incubated for
30 minutes at $37^{\circ} \mathrm{C}$. Absorbance at $490 \mathrm{~nm}$ was read using a microplate reader (Spectramax M3; Molecular Devices). Cells treated or not treated with $10 \%$ Triton X-100 were used as positive control and negative control, respectively.

Measurement of 20S Proteasome Activity. Proteasome activity was assayed using 20S proteasome assay kit (Cayman Chemical) according to the manufacturer's protocol. After treatment with MG132, bortezomib, or carfilzomib, hOAT1 cells were washed with $200 \mu \mathrm{l}$ of assay buffer and lysed with $100 \mu \mathrm{l}$ of lysis buffer. Then, $90 \mu \mathrm{l}$ of supernatant was transferred to a black 96-well plate, and $10 \mu \mathrm{l}$ of assay buffer and $10 \mu \mathrm{l}$ of substrate solution (SUC-LLVY-AMC) were added and incubated for 1 hour at $37^{\circ} \mathrm{C}$. Fluorescence intensity (excitation $360 \mathrm{~nm}$, emission $480 \mathrm{~nm}$ ) was measured using a microplate reader (Spectramax M3; Molecular Devices).

Cell Surface Biotinylation. The cell surface expression level of hOAT1 was examined using the method published in our laboratory (Zhang et al., 2013). hOAT1 cells were incubated with $1 \mathrm{ml}$ of freshly made sulfo-NHS-SS-biotin $(0.5 \mathrm{mg} / \mathrm{ml}$ in $\mathrm{PBS} / \mathrm{CM})$ in two successive 20 -minute incubations on ice with very gentle shaking. After biotinylation, each dish was briefly rinsed with $3 \mathrm{ml}$ of $\mathrm{PBS} / \mathrm{CM}$ containing $100 \mathrm{mM}$ glycine and then incubated with the same solution for 20 minutes on ice to ensure complete quenching of the unreacted sulfo-NHS-SS-biotin. The cells were then lysed on ice for 50 minutes in $400 \mu \mathrm{l}$ of lysis buffer (10 mM Tris-HCl, $\mathrm{pH} 7.5$, $150 \mathrm{mM} \mathrm{NaCl}, 1 \mathrm{mM}$ EDTA, 0.1\% SDS, 1\% Triton X-100) with $1 \%$ proteinase inhibitor cocktail. The cell lysates were cleared by centrifugation at $16,000 \mathrm{~g}$ at $4^{\circ} \mathrm{C}$. Streptavidin-agarose resin $(40 \mu \mathrm{l})$ was then added to the supernatant to isolate cell membrane proteins. hOAT1 was detected in the pool of surface proteins by SDS-PAGE and immunoblotting using anti-Myc antibody 9E10.

Immunoprecipitation. The ubiquitination of hOAT1 was detected using the immunoprecipitation method published in our laboratory (Zhang et al., 2013). hOAT1 cells were lysed with lysis buffer $(50 \mathrm{mM}$ Tris-HCl, pH 8.0, $150 \mathrm{mM} \mathrm{NaCl}, 1 \%$ Triton X-100, $10 \%$ glycerol, $5 \mathrm{mM}$ EDTA, and $1 \mathrm{mM} \mathrm{NaF}$ ) with $1 \%$ proteinase inhibitor cocktail and $20 \mathrm{mM} N$-ethylmaleimide. Cell lysates were precleared with protein $\mathrm{G}$-agarose to reduce nonspecific binding at $4^{\circ} \mathrm{C}$ for 2 hours. Anti-Myc antibody was incubated with $30 \mu \mathrm{l}$ of protein G-agarose at $4^{\circ} \mathrm{C}$ for 2 hours. The precleared protein sample was then mixed with antibodybound protein G-agarose and underwent end-over-end rotating at $4{ }^{\circ} \mathrm{C}$ overnight. Proteins bound to the protein G-agarose were eluted with urea buffer containing $\beta$-mecaptoethanol and analyzed by immunoblotting with indicated antibodies.

Degradation Assay. The degradation of hOAT1 was assayed using the method published in our laboratory (Wang et al., 2016). hOAT1 cells were first biotinylated, and then the labeled cells were incubated in DMEM with or without bortezomib and carfilzomib at $37^{\circ} \mathrm{C}$. Treated cells were collected at $0,4,8$, and 12 hours and lysed in lysis buffer with $1 \%$ protease inhibitor cocktail. The cell lysates were cleared by centrifugation at $16,000 \mathrm{~g}$ at $4^{\circ} \mathrm{C}$. Streptavidin-agarose resin $(40 \mu \mathrm{l})$ was then added to the supernatant to isolate cell membrane proteins. Samples were loaded on 7.5\% SDS-PAGE minigels and analyzed by immunoblotting with anti-Myc antibody.

Electrophoresis and Immunoblotting. Electrophoresis and immunoblotting were performed using the method published in our laboratory (Zhang et al., 2008). Protein samples were resolved on 7.5\% SDS-PAGE minigels and electroblotted onto polyvinylidene difluoride membranes. The blots were blocked for 1 hour with $5 \%$ nonfat dry milk in PBS- $0.05 \%$ Tween 20 , washed, and incubated overnight at $4^{\circ} \mathrm{C}$ with appropriate primary antibodies, followed by horseradish peroxidase-conjugated secondary antibodies. The signals were detected by SuperSignal West Dura Extended Duration Substrate kit (Thermo Scientific). Nonsaturating immunoreactive protein bands were quantified by scanning densitometry with the FluorChem 8000 imaging system (Alpha Innotech Corp., San Leandro, CA).

Data Analysis. Each experiment was repeated a minimum of three times. The statistical analysis was from multiple experiments. Among multiple treatments, a one-way ANOVA or two-way ANOVA 
Tukey's test was applied by using GraphPad Prism software (GraphPad Software Inc., San Diego, CA). A $P$ value of $<0.05$ was considered statistically significant.

\section{Results}

Effects of Protease Inhibitors on the Accumulation of Ubiquitinated OAT1. To determine the intracellular degradation system for ubiquitinated OAT1, we treated OAT1-expressing cells with proteasome inhibitors MG132 or ALLN and lysosome inhibitors leupeptin or pepstatin A for 2 hours. The treated cells were lysed, and OAT1 was pulled down by anti-Myc antibody (epitope Myc was tagged to OAT1 to facilitate immunodetection) or with mouse IgG (as negative control), followed by immunoblotting (IB) with anti-ubiquitin antibody (anti-Ub) to detect ubiquitinated OAT1. Our results (Fig. 1, top panel) revealed that although incubation of the cells with the lysosomal inhibitors leupeptin and pepstatin A was without any effect, incubation with proteasomal inhibitors MG132 and ALLN both led to a substantial accumulation of ubiquitinated OAT1, suggesting that ubiquitinated OAT1 degrades through proteasomes rather than lysosomes. The change of ubiquitinated OAT1 did not result from the difference of OAT1 immunoprecipitated because the amount of OAT1 pulled down was similar among all samples (Fig. 1, bottom panel).

Effects of Proteasomal Inhibitors Bortezomib and Carfilzomib on the Accumulation of Ubiquitinated OAT1. Bortezomib and carfilzomib are Food and Drug Administration-approved anticancer drugs acting as selective proteasome inhibitors. We therefore examined the effects of bortezomib and carfilzomib on the accumulation of ubiquitinated OAT1. OAT1-expressing cells were treated with bortezomib or carfilzomib for 12 hours. The treated cells were lysed, and OAT1 was pulled down by anti-Myc antibody, followed by immunoblotting (IB) with anti-Ub to detect ubiquitinated OAT1. Our results (Fig. 2, top panel) revealed that incubation of the cells with bortezomib or carfilzomib led to a substantial accumulation of ubiquitinated OAT1. The change of ubiquitinated OAT1 did not result from the difference of OAT1 immunoprecipitated because the amount of OAT1 pulled down was similar among all samples (Fig. 2, bottom panel; the full blot is included in Supplemental Fig. 1).

Cis-Effect of Bortezomib or Carfilzomib on OAT1-Mediated Uptake of $\left[{ }^{\mathbf{3}} \mathbf{H}\right]$ p-Aminohippuric Acid. As OAT 1 is a multisubstrate transporter, we examined whether bortezomib and carfilzomib are inhibitors for OAT1 by carrying out a cis-inhibition experiment (Fig. 3). We measured a 3-minute uptake of $\left[{ }^{3} \mathrm{H}\right] \mathrm{PAH}(20 \mu \mathrm{M})$ into OAT1-expressing cells with $20 \mu \mathrm{M}$ probenecid, $3 \mu \mathrm{M}$ bortezomib, or carfilzomib being present in the same solution as PAH. Probenecid is a well-known competitive inhibitor for OAT (Vallon et al., 2012; Wang et al., 2014; Nigam et al., 2015). Under such conditions, probenecid inhibited OAT1-mediated transport of $\left[{ }^{3} \mathrm{H}\right] \mathrm{PAH}$ by $41 \%$ [95\% confidence interval (CI): 36\%-46\%], whereas bortezomib and carfilzomib were without any effect, suggesting that bortezomib and carfilzomib are not inhibitors for OAT1. Therefore, bortezomib and carfilzomib do not regulate OAT1 through its ability to interfere with the transporter.

Effects of Bortezomib and Carfilzomib on OAT1-Mediated Uptake of p-Aminohippuric Acid. OAT1-expressing cells were treated with bortezomib and carfilzomib for 12 hours,

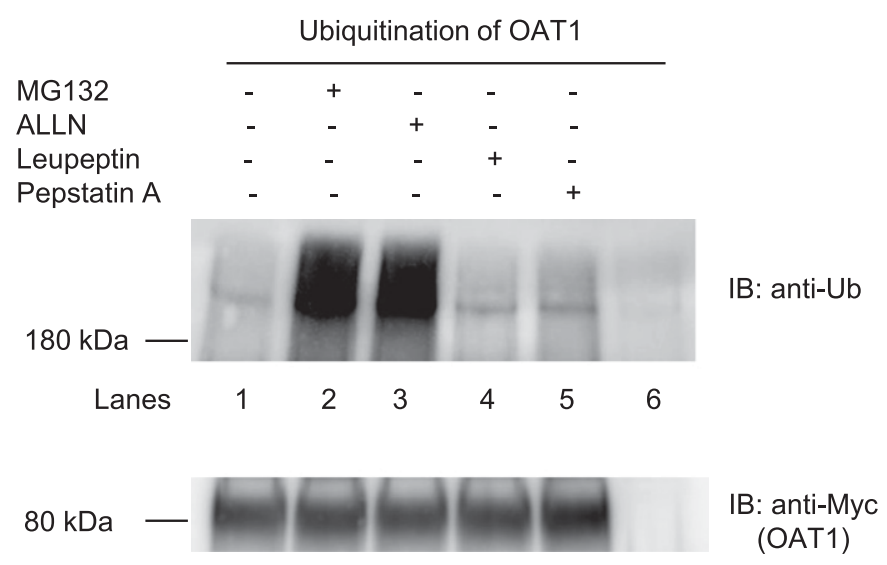

Fig. 1. Effects of protease inhibitors on the accumulation of ubiquitinated OAT1. Top panel: OAT1-expressing HEK293 cells were treated with proteasome inhibitors MG132 $(10 \mu \mathrm{M})$ or ALLN $(100 \mu \mathrm{M})$ or lysosome inhibitors leupeptin $(10 \mu \mathrm{M})$ or pepstatin $\mathrm{A}(10 \mu \mathrm{M})$ for 2 hours. Treated cells were then lysed, and OAT1 was immunoprecipitated with anti-Myc antibody or with mouse IgG (as negative control, lane 6), followed by IB with anti-Ub. Bottom panel: the same immunoblot from the top panel was reprobed with anti-Myc antibody to determine the amount of OAT1 immunoprecipitated.

and then cytotoxicity and OAT1-mediated uptake of PAH were measured. Both bortezomib and carfilzomib induced stimulation of PAH uptake at $25-100 \mathrm{nM}$ for bortezomib (Fig. 4A) and 0.1-1 $\mu \mathrm{M}$ for carfilzomib (Fig. 4B) without cytotoxicity at corresponding concentration (Fig. 5). The transport activity of OAT1 was increased by $11 \%(95 \% \mathrm{CI}:-7 \%$ to $30 \%)$ and $49 \%(95 \%$ CI: $31 \%-67 \%)$, respectively, at 5 and $25 \mathrm{nM}$ bortezomib. In contrast, 12 hours of treatment with lysosome inhibitors leupeptin and pepstatin A did not affect the uptake of PAH (Fig. 4C). Further study showed that, like MG132, bortezomib and carfilzomib inhibited the $20 \mathrm{~S}$ proteasome activity after 2 hours of treatment (Fig. 6A). For bortezomib at 1,5 , and $25 \mathrm{nM}$ with treatment for 12 hours, the proteasome activity was inhibited by $8 \%$ (95\% CI: $5 \%-12 \%), 29 \%$ (95\% CI: 26\%-33\%), and 87\% (95\% CI: 84\%-91\%), respectively, which

\begin{tabular}{lllllll} 
& \multicolumn{3}{c}{ Ubiquitination of OAT1 } \\
\cline { 2 - 6 } Bortezomib & - & + & & - & - \\
Carfilzomib & - & - & - & +
\end{tabular}
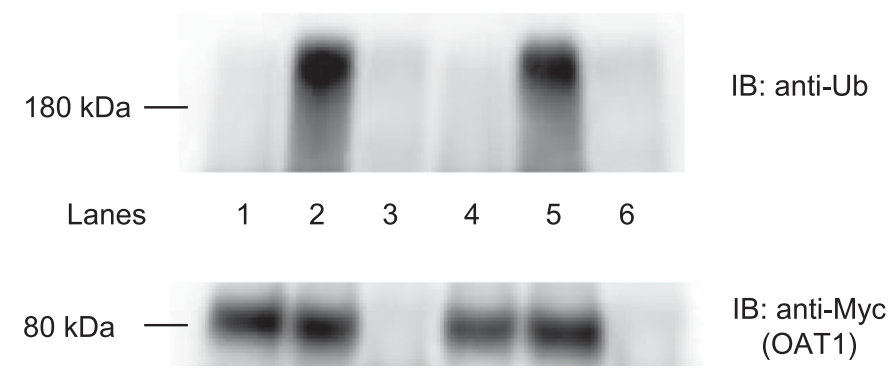

Fig. 2. Effects of proteasomal inhibitors bortezomib and carfilzomib on the accumulation of ubiquitinated OAT1. Top panel: OAT1-expressing HEK293 cells were treated with bortezomib (25 nM) or carfilzomib $(0.5 \mu \mathrm{M})$ for 12 hours. Treated cells were then lysed, and OAT1 was immunoprecipitated with anti-Myc antibody or mouse IgG (as negative control, lane 3 and 6), followed by IB with anti-Ub. Bottom panel: the same immunoblot from the top panel was reprobed with anti-Myc antibody to determine the amount of OAT1 immunoprecipitated. 


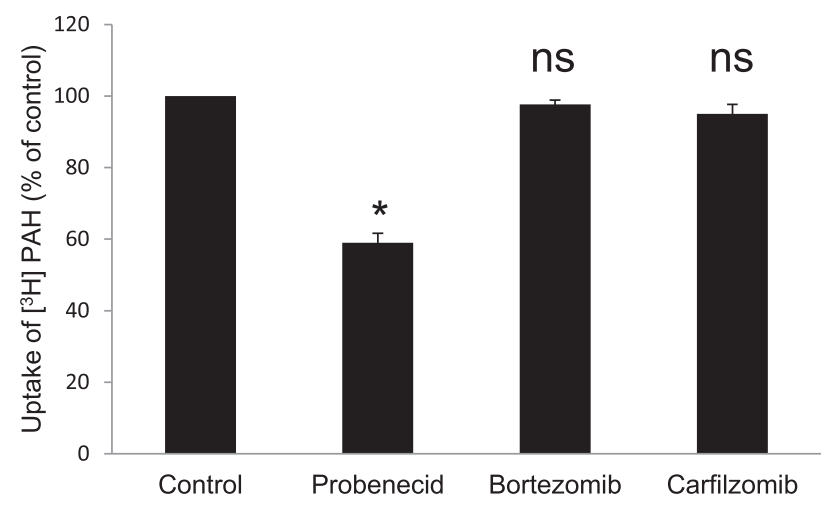

Fig. 3. Cis-effect of bortezomib or carfilzomib on OAT1-mediated uptake of $\left[{ }^{3} \mathrm{H}\right] \mathrm{PAH}$. The uptake of $\left[{ }^{3} \mathrm{H}\right] \mathrm{PAH}(20 \mu \mathrm{M})$ in the presence of bortezomib $(3 \mu \mathrm{M})$, carfilzomib $(3 \mu \mathrm{M})$, or probenecid $(20 \mu \mathrm{M})$ for 3 minutes was measured in OAT1-expressing HEK293 cells. Each data point represented only carrier-mediated transport after subtraction of values from parental cells. Uptake activity was expressed as the percentage of uptake measured in control cells from three independent experiments. Values are means \pm S.D. $(n=3) .{ }^{*} P<0.05 ; \mathrm{ns}$, not statistically significant.

showed a concentration-dependent inhibition of proteasome activity at 1-25 nM bortezomib (Fig. 6B).

Effect of Bortezomib and Carfilzomib on OAT1 Expression. OAT1-expressing cells were treated with bortezomib or carfilzomib, and OAT1 expression both at the cell surface and in the total cell lysates was examined. We showed that treatment with bortezomib or carfilzomib led to an increase of OAT1 expression at the cell surface (Fig. 7A, top panel, and Fig. 7B) and in total cell lysate (Fig. 7C, top panel, Fig. 7D). Such a change in OAT1 expression was not due to the general perturbation of cellular proteins, as the expression of cell surface membrane protein marker E-Cadherin (Fig. 7A, bottom panel) and cellular protein marker GAPDH (Fig. 7C, bottom panel) was not affected under these conditions.
Effect of Bortezomib and Carfilzomib on OAT1 Stability. The stability of cell surface OAT1 was subsequently assessed based on a biotinylation approach. OAT1-expressing cells were biotinylated with membrane-impermeable biotinylation reagent sulfo-NHS-SS-biotin. Labeled cells were treated with or without bortezomib or carfilzomib for 12 hours. Treated cells were then lysed, and cell surface proteins were isolated using streptavidin-agarose resin, followed by immunoblotting with anti-Myc antibody. Our results (Fig. 8) showed that the rate of OAT1 degradation decreased substantially after 8 and 12 hours of treatment with bortezomib (Fig. 8, A and B) or carfilzomib (Fig. 8, C and D) as compared with that of control. These results indicate that bortezomib and carfilzomib substantially increase OAT1 stability.

\section{Discussion}

The transport activity of OAT1 is critically dependent on its expression level at the cell surface. We previously demonstrated that post-translational modification of OAT1 by ubiquitination leads to OAT1 internalization from the cell surface and subsequent degradation in proteolytic systems (Zhang et al., 2008, 2013; Xu et al., 2017). In the current study, we identified the proteolytic system in which the ubiquitinated OAT1 is degraded and revealed a new strategy of reversing ubiquitination-dependent OAT1 degradation.

HEK293 cells, a heterologous cell system, was used in our current studies, as they have been widely used for research in the regulation of the cloned organic anion transporters and other renal transporters (Rodiger et al., 2010; Xue et al., 2011; Zeng et al., 2012; El-Sheikh et al., 2013). The fact that these cells do not express endogenous OATs is particularly advantageous because expression of OAT1 in these cells will permit us to analyze the transport properties of OAT1 without being disturbed by other organic anion transporters. Our studies in HEK293 cells will pave the path for the upcoming work
A

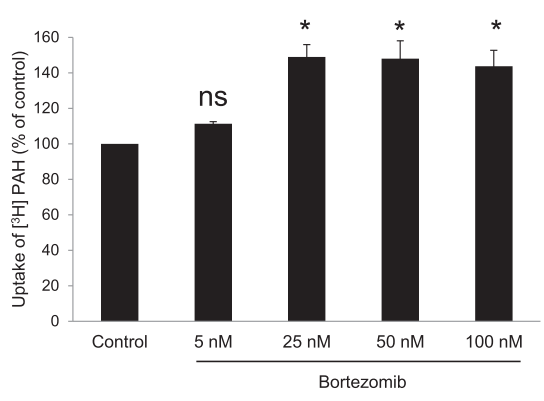

B

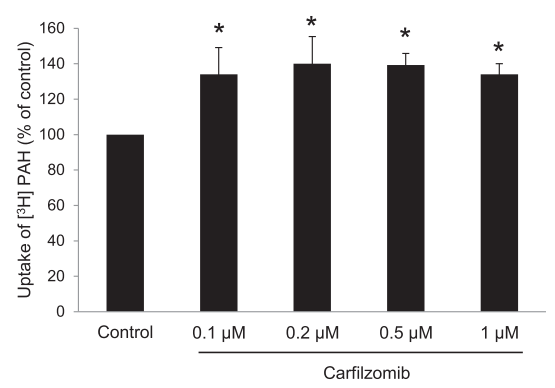

C

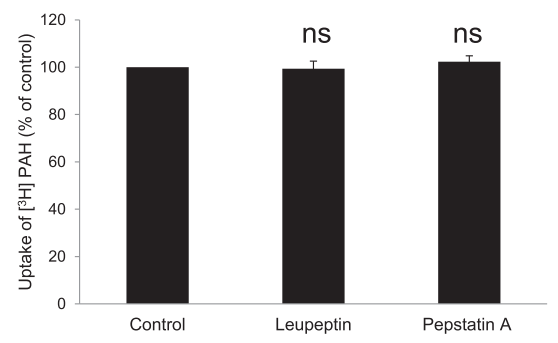

Fig. 4. Effect of bortezomib, carfilzomib, and lysosome inhibitors on OAT1 activity. OAT1-expressing HEK293 cells were treated with bortezomib (A) or with carfilzomib (B) at indicated concentrations or lysosome inhibitors leupeptin $(10 \mu \mathrm{M})$ or pepstatin $\mathrm{A}(10 \mu \mathrm{M})(\mathrm{C})$ for 12 hours. The uptake of $\left[{ }^{3} \mathrm{H}\right] \mathrm{PAH}(20 \mu \mathrm{M})$ for 3 minutes was then performed. Each data point represented only carrier-mediated transport after subtraction of values from parental cells. Uptake activity was expressed as the percentage of uptake measured in control cells from three independent experiments. Values are means \pm S.D. $(n=3) . * P<0.05$. ns, not statistically significant. 
A

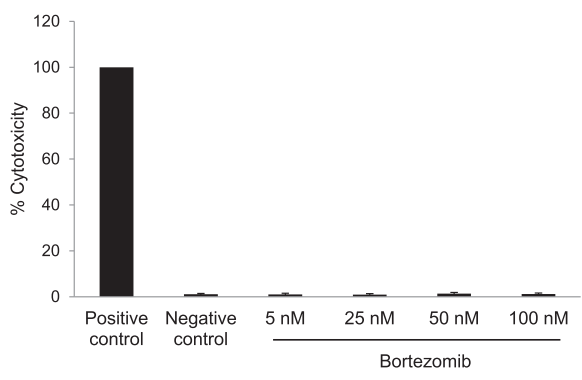

B

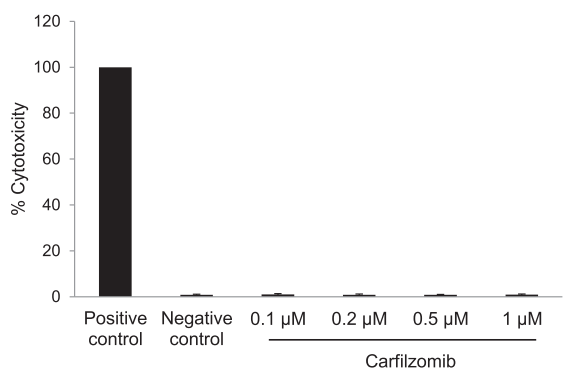

Fig. 5. Cytotoxicity of bortezomib and carfilzomib on OAT1-expressing HEK293 cells. OAT1-expressing HEK293 cells were treated with bortezomib (A) or with carfilzomib (B) at indicated concentrations for 12 hours. The $\mathrm{LDH}$ released in supernatant was then performed. Cells treated or not treated with $10 \%$ Triton X-100 were used as positive control and negative control, respectively. focusing on validating whether the same mechanisms exist in primary epithelia.

Our current study unveiled a key role for proteasomes in the regulation of OAT1 expression and transport activity. Treatment of OAT1-expressing cells with proteasomal inhibitors MG132 and ALLN led to an accumulation of ubiquitinated OAT1, whereas treatment of cells with lysosomal inhibitors leupeptin and pepstatin A were without any effect (Fig. 1). The degradation of cell surface OAT1 through proteasomes contrasted with most of the plasma membrane proteins, for which the canonical degradation pathway is through lysosomes (Piper and Luzio, 2007; Varghese et al., 2008). This finding opens the door for new strategies in modulating OAT1 function through controlling proteasomal activity.

Proteasomal inhibition has been developed for antitumor activities. The proteasomal inhibitors used in our current studies, bortezomib and carfilzomib, are two Food and Drug Administration-approved antitumor drugs with the ability to inhibit proteasomes. Bortezomib administration caused a decrease of $20 \mathrm{~S}$ proteasome activity in the white blood cells, liver, colon, muscle, and prostate (Adams et al., 1999; Bross et al., 2004). Inhibition of the $20 \mathrm{~S}$ proteasome activity after carfilzomib administration was observed in blood, adrenal, heart, lung, spleen, bone marrow, and kidney (Nooka et al., 2013). Both drugs have also been shown to affect kidney function. Bortezomib can attenuate renal impairment in patients with multiple myeloma, renal fibrosis, and lupus nephritis (Hainz et al., 2012; Ward et al., 2012; Zeniya et al., 2017; Zhang et al., 2017; Cohen et al., 2015; Dimopoulos et al., 2016). Carfilzomib inhibited the chymotrypsin-like activity of the $20 \mathrm{~S}$ proteasome in the kidney by $50 \%-60 \%$ in rats (https://www.accessdata.fda.gov/drugsatfda_docs/nda/2012/ 202714Orig1s000PharmR.pdf). Recent studies showed that bortezomib can affect the activities of copper transporter 1 ,
ATP-binding cassette transporter A1 and ATP-binding cassette transporter G1, metal transporter Zrt/IRT-like protein 14 , and organic anion transporting polypeptide 1B3 (Jandial et al., 2009; Ogura et al., 2011; Zhao et al., 2014; Alam et al., 2017). However, the potential of these antitumor drugs to modulate OAT1 activity has not been explored. By inhibiting the proteasomal activity, these drugs gained a new role in the regulation of OAT1 activity.

We showed that treatment of OAT1-expressing cells with bortezomib and carfilzomib led to a substantial accumulation of the ubiquitinated OAT1 (Fig. 2), which correlated well with an enhanced OAT1 transport activity and an enhanced OAT1 expression at the cell surface (Figs. 4 and 7). The molecular weight of ubiquitinated OAT1 was more than $180 \mathrm{kDa}$, $\sim 100 \mathrm{kDa}$ larger than unubiquitinated OAT1 ( $~ 80 \mathrm{kDa})$. Since ubiquitin is an $8-\mathrm{kDa}$ polypeptide, OAT1 may be poly- or multiubiquitinated (Figs. 1 and 2).

The increase of surface OAT1 can be attributed to reduced internalization, increased recycling, or decreased degradation. As internalization and recycling are rapid processes, the alteration of internalization or recycling may be the mechanisms in acute regulation of OAT1 during the short term ( $<30$ minutes), whereas the alteration of degradation may be the mechanism in chronic regulation of OAT1 during the long term (several hours) (Zhang et al., 2008, 2012, 2013; Xu et al., 2017; Wang et al., 2019). Bortezomib and carfilzomib enhanced the surface expression and transport activity of OAT1 with 12 hours of treatment and did not stimulate the transport activity in short-term treatment, suggesting that reduced internalization or increased recycling were not involved in OAT1 regulation. Further exploring the underlying mechanism, we found that the degradation rate of OAT1 was decelerated after treatment with bortezomib and carfilzomib (Fig. 8). The drug target of bortezomib and carfilzomib is $20 \mathrm{~S}$
A

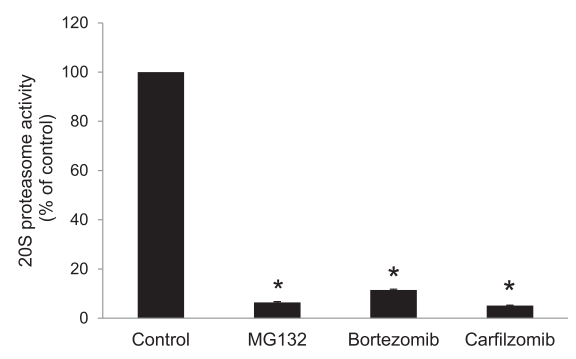

B

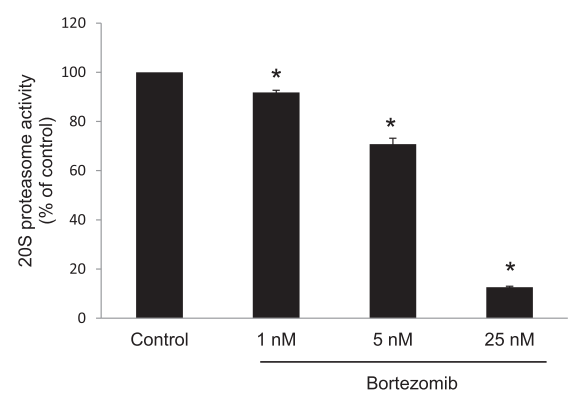

Fig. 6. Effect of bortezomib and carfilzomib on the $20 \mathrm{~S}$ proteasome activity. (A) OAT1-expressing HEK293 cells were treated with MG132 $(10 \mu \mathrm{M})$, bortezomib (25 nM), or carfilzomib $(0.5 \mu \mathrm{M})$ for 2 hours. (B) OAT1expressing HEK293 cells were treated with bortezomib (1, 5, and $25 \mathrm{nM})$ for 12 hours. The 20S proteasome activity of cells was then performed. The $20 \mathrm{~S}$ proteasome activity was expressed as the percentage of control cells from three independent experiments. Values are means \pm S.D. $(n=3) .{ }^{*} P<0.05$. 
A

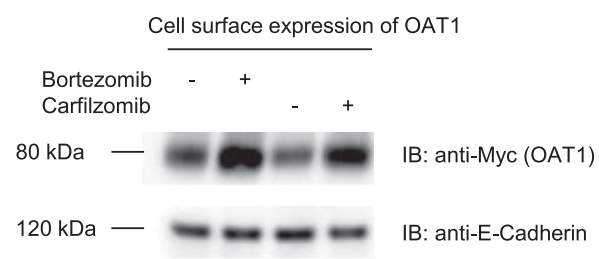

C

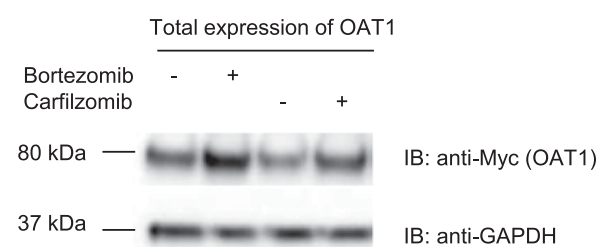

B

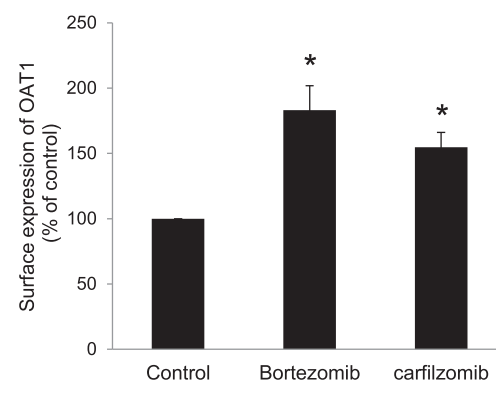

D

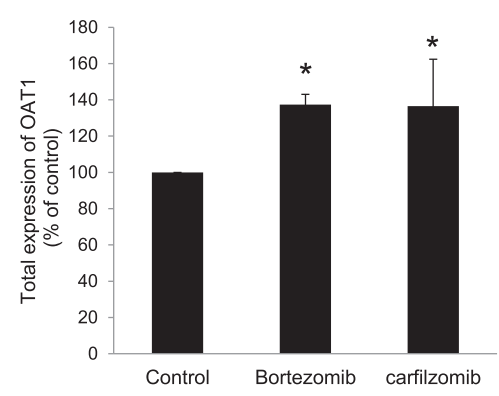

Fig. 7. Effect of bortezomib and carfilzomib on OAT1 expression. (A) Top panel: OAT1-expressing HEK293 cells were treated with bortezomib $(25 \mathrm{nM})$ or carfilzomib $(0.5 \mu \mathrm{M})$ for 12 hours. Cell surface biotinylation was performed. Biotinylated (cell surface) proteins were separated with using streptavidin-agarose resin and analyzed by IB with an anti-Myc antibody. Bottom panel: the same blot from the top panel was reprobed with an anti-E-Cadherin antibody. E-Cadherin is an integral membrane protein marker. (B) Densitometry plot of results from (A), top panel, as well as from other experiments. Values are means \pm S.D. $(n=3) .{ }^{*} P<0.05$. (C) Top panel: OAT1-expressing HEK293 cells were treated with bortezomib $(25 \mathrm{nM})$ or carfilzomib $(0.5 \mu \mathrm{M})$ for 12 hours. Cells were then lysed, followed by IB with antiMyc antibody. Bottom panel: the same blot from the top panel was reprobed with an anti-GAPDH antibody. GAPDH is a cellular protein marker. (D) Densitometry plot of results from (C), top panel, as well as from other experiments. Values are means \pm S.D. $(n=3) . * P<0.05$ proteasome. Our results showed that bortezomib and carfilzomib inhibited $20 \mathrm{~S}$ proteasome activity (Fig. 6A), and there was a correlation between the degree of proteasomal inhibition and increase of OAT1 transporter function at 5 and $25 \mathrm{nM}$ bortezomib (Fig. 4A; Fig. 6B). Therefore, bortezomib- and carfilzomib-stimulated OAT1 expression and transport activity was mainly due to proteasome inhibition and the subsequent deceleration rate of OAT1 degradation.

After intravenous administration of the first $1.3-\mathrm{mg} / \mathrm{m}^{2}$ dose to multiple myeloma patients, the mean maximum plasma concentration $\left(\mathrm{C}_{\max }\right)$ of bortezomib was $291 \mathrm{nM}(112 \mathrm{ng} / \mathrm{ml}$ ) (Reece et al., 2011). For carfilzomib, the mean $C_{\max }$ values after a 2- to 10-minute intravenous infusion of a $27-\mathrm{mg} / \mathrm{m}^{2}$ dose or a 30 -minute infusion of a $56-\mathrm{mg} / \mathrm{m}^{2}$ dose were $5.9(4232 \mathrm{ng} / \mathrm{ml})$ and $2.9 \mu \mathrm{M}(2079 \mathrm{ng} / \mathrm{ml})$, respectively (https://www.accessdata.fda.gov/drugsatfda_docs/ label/2018/202714s019lbl.pdf). The concentration of bortezomib (5-100 nM) and carfilzomin $(0.1-1 \mu \mathrm{M})$ we selected in the cell model has a clinical relevance. Besides, the influence of other proteasome inhibitors, such as ixazomib (approved), marizomib, oprozomib, and delanzomib (in clinical trials), on the kidney OAT1 should be given attention. In this study, we reported the cellular mechanisms, and an in vivo study is currently underway in our laboratory to validate the roles of bortezomib or carfilzomib in OTA1 ubiquitination, expression, and renal clearance of drugs.

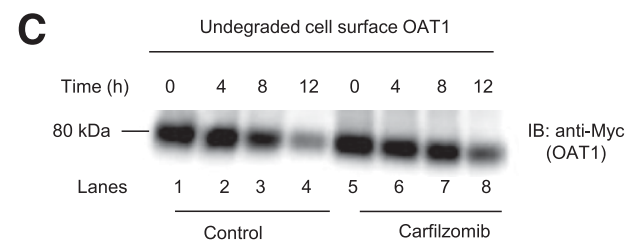

D

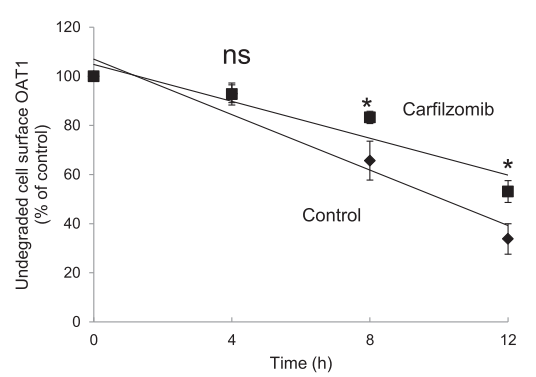

Fig. 8. Effect of bortezomib or carfilzomib on OAT1 stability. (A) OAT1-expressing HEK293 cells were biotinylated with membrane-impermeable biotinylation reagent sulfo-NHS-SS-biotin. Labeled cells were then treated with bortezomib $(25 \mathrm{nM})$ at $37^{\circ} \mathrm{C}$ for 4,8 , and 12 hours, respectively. Treated cells were lysed, and cell surface proteins were isolated using streptavidin-agarose resin, followed by IB with anti-Myc antibody. (B) Densitometry plot of results from (A) as well as from other experiments. Values are means \pm S.D. $(n=3)$. ${ }^{*} P<0.05$. (C) OAT1-expressing HEK293 cells were biotinylated with membrane-impermeable biotinylation reagent sulfo-NHS-SS-biotin. Labeled cells were then treated with carfilzomib $(0.5 \mu \mathrm{M})$ at $37^{\circ} \mathrm{C}$ for $4,8,12$ hours, respectively. Treated cells were lysed, and cell surface proteins were isolated using streptavidin-agarose resin, followed by IB with anti-Myc antibody. (D) Densitometry plot of results from (C) as well as from other experiments. Values are means \pm S.D. $(n=3)$. $* P<0.05$; ns, not statistically significant. 
Like proteasomes, several deubiquitinases may also regulate the OAT function. These deubiquitinases are associated with the 19S regulatory particles of proteasome (D'Arcy et al., 2015). Recently, it has been revealed that inhibition of proteasome-associated deubiquitinase activity is an alternative strategy to $20 \mathrm{~S}$ proteasome inhibitors for cancer treatment (Chen et al., 2017; Mofers et al., 2017). Therefore, it is interesting to explore whether proteasome-associated deubiquitinase is a novel target for OAT1 regulation.

Our studies showing that proteasome inhibitors, e.g., bortezomib and carfilzomib, can stimulate the transport activity of OAT1 has physiologic implications. When drugs are overdose or endogenous/exogenous metabolites, uremic/environmental toxins are increased in blood, and we can use this method to accelerate their clearance to avoid systemic toxicity and maintain the body's homeostasis. It can also be used to reverse the decreased expression under disease conditions.

In conclusion, our study demonstrated for the first time that ubiquitinated cell surface OAT1 degrades through proteasome instead of lysosome, and anticancer drugs bortezomib and carfilzomib have a novel role in regulating OAT1 expression and transport activity, indicating their potential influence on the OAT1-mediated renal excretion of drugs during cancer and comorbidity therapies.

\section{Acknowledgments}

We thank Haoxun Wang for his preliminary work, which paved the way for this study.

\section{Authorship Contributions}

Participated in research design: Fan, You.

Conducted experiments: Fan.

Performed data analysis: Fan, You.

Wrote or contributed to the writing of the manuscript: Fan, You.

\section{References}

Adams J, Palombella VJ, Sausville EA, Johnson J, Destree A, Lazarus DD, Maas J, Pien CS, Prakash S, and Elliott PJ (1999) Proteasome inhibitors: a novel class of potent and effective antitumor agents. Cancer Res 59:2615-2622.

Alam K, Farasyn T, Crowe A, Ding K, and Yue W (2017) Treatment with proteasome inhibitor bortezomib decreases organic anion transporting polypeptide (OATP) 1B3-mediated transport in a substrate-dependent manner. PLoS One 12:e0186924.

Brandoni A, Anzai N, Kanai Y, Endou H, and Torres AM (2006a) Renal elimination of p-aminohippurate $(\mathrm{PAH})$ in response to three days of biliary obstruction in the rat. The role of OAT1 and OAT3. Biochim Biophys Acta 1762:673-682.

Brandoni A, Villar SR, Picena JC, Anzai N, Endou H, and Torres AM (2006b) Expression of rat renal cortical OAT1 and OAT3 in response to acute biliary obstruction. Hepatology 43:1092-1100.

Bross PF, Kane R, Farrell AT, Abraham S, Benson K, Brower ME, Bradley S, Gobburu JV, Goheer A, Lee SL, et al. (2004) Approval summary for bortezomib for injection in the treatment of multiple myeloma. Clin Cancer Res 10(12 Pt 1):3954-3964.

Burckhardt G (2012) Drug transport by Organic Anion Transporters (OATs). Pharmacol Ther 136:106-130.

Chen X, Yang Q, Xiao L, Tang D, Dou QP, and Liu J (2017) Metal-based proteasomal deubiquitinase inhibitors as potential anticancer agents. Cancer Metastasis Rev 36: 655-668.

Clague MJ and Urbé S (2010) Ubiquitin: same molecule, different degradation pathways. Cell 143:682-685.

Cohen C, Royer B, Javaugue V, Szalat R, El Karoui K, Caulier A, Knebelmann B, Jaccard A, Chevret S, Touchard G, et al. (2015) Bortezomib produces high hematological response rates with prolonged renal survival in monoclonal immunoglobulin deposition disease. Kidney Int 88:1135-1143.

D'Arcy P, Wang X, and Linder S (2015) Deubiquitinase inhibition as a cancer therapeutic strategy. Pharmacol Ther 147:32-54.

Dimopoulos MA, Sonneveld P, Leung N, Merlini G, Ludwig H, Kastritis E, Goldschmidt H, Joshua D, Orlowski RZ, Powles R, et al. (2016) International myeloma working group recommendations for the diagnosis and management of myelomarelated renal impairment. J Clin Oncol 34:1544-1557.

El-Sheikh AA, Greupink R, Wortelboer HM, van den Heuvel JJ, Schreurs M, Koenderink JB, Masereeuw R, and Russel FG (2013) Interaction of immunosuppressive drugs with human organic anion transporter (OAT) 1 and OAT3, and multidrug resistanceassociated protein (MRP) 2 and MRP4. Transl Res 162:398-409.

Goldberg AL and Rock K (2002) Not just research tools--proteasome inhibitors offer therapeutic promise. Nat Med 8:338-340.
Hainz N, Thomas S, Neubert K, Meister S, Benz K, Rauh M, Daniel C, Wiesener M, Voll RE, and Amann K (2012) The proteasome inhibitor bortezomib prevents lupus nephritis in the NZB/W F1 mouse model by preservation of glomerular and tubulointerstitial architecture. Nephron, Exp Nephrol 120:e47-e58.

Jandial DD, Farshchi-Heydari S, Larson CA, Elliott GI, Wrasidlo WJ, and Howell SB (2009) Enhanced delivery of cisplatin to intraperitoneal ovarian carcinomas mediated by the effects of bortezomib on the human copper transporter 1. Clin Cancer Res 15(2):553-560.

Kim GH, Na KY, Kim SY, Joo KW, Oh YK, Chae SW, Endou H, and Han JS (2003) Up-regulation of organic anion transporter 1 protein is induced by chronic furosemide or hydrochlorothiazide infusion in rat kidney. Nephrol Dial Transplant 18: $1505-1511$.

Kisselev AF and Goldberg AL (2001) Proteasome inhibitors: from research tools to drug candidates. Chem Biol 8:739-758.

Kwon O, Hong SM, and Blouch K (2007) Alteration in renal organic anion transporter 1 after ischemia/reperfusion in cadaveric renal allografts. $J$ Histochem $C y$ tochem 55:575-584.

Kwon O, Wang WW, and Miller S (2008) Renal organic anion transporter 1 is maldistributed and diminishes in proximal tubule cells but increases in vasculature after ischemia and reperfusion. Am J Physiol Renal Physiol 295 : F1807-F1816.

Lee S, Sato Y, and Nixon RA (2011) Lysosomal proteolysis inhibition selectively disrupts axonal transport of degradative organelles and causes an Alzheimer's-like axonal dystrophy. $J$ Neurosci 31:7817-7830.

Liang Y, Li S, and Chen L (2015) The physiological role of drug transporters. Protein Cell 6:334-350.

Miao Q, Liu Q, Wang C, Meng Q, Guo X, Sun H, Peng J, Ma X, Kaku T, and Liu K (2013) Inhibitory effect of $1 \alpha, 25$-dihydroxyvitamin $\mathrm{D}_{3}$ on excretion of JBP485 via organic anion transporters in rats. Eur.J Pharm Sci 48:351-359.

Mofers A, Pellegrini P, Linder S, and D'Arcy P (2017) Proteasome-associated deubiquitinases and cancer. Cancer Metastasis Rev 36:635-653.

Mónica Torres A, Mac Laughlin M, Muller A, Brandoni A, Anzai N, and Endou H (2005) Altered renal elimination of organic anions in rats with chronic renal failure. Biochim Biophys Acta 1740:29-37.

Nigam SK (2015) What do drug transporters really do? Nat Rev Drug Discov 14: 29-44

Nigam SK, Bush KT, Martovetsky G, Ahn SY, Liu HC, Richard E, Bhatnagar V, and $\mathrm{Wu} \mathrm{W}$ (2015) The organic anion transporter (OAT) family: a systems biology perspective. Physiol Rev 95:83-123.

Nooka A, Gleason C, Casbourne D, and Lonial S (2013) Relapsed and refractory lymphoid neoplasms and multiple myeloma with a focus on carfilzomib. Biologics 7:13-32.

Ogura M, Ayaori M, Terao Y, Hisada T, Iizuka M, Takiguchi S, Uto-Kondo H, Yakushiji E, Nakaya K, Sasaki M, et al. (2011) Proteasomal inhibition promotes ATP-binding cassette transporter A1 (ABCA1) and ABCG1 expression and cholesterol efflux from macrophages in vitro and in vivo. Arterioscler Thromb Vasc Biol 31:1980-1987.

Piper RC and Luzio JP (2007) Ubiquitin-dependent sorting of integral membrane proteins for degradation in lysosomes. Curr Opin Cell Biol 19:459-465.

Reece DE, Sullivan D, Lonial S, Mohrbacher AF, Chatta G, Shustik C, Burris H III, Venkatakrishnan K, Neuwirth R, Riordan WJ, et al. (2011) Pharmacokinetic and pharmacodynamic study of two doses of bortezomib in patients with relapsed multiple myeloma. Cancer Chemother Pharmacol 67:57-67.

Rödiger M, Zhang X, Ugele B, Gersdorff N, Wright SH, Burckhardt G, and Bahn A (2010) Organic anion transporter 3 (OAT3) and renal transport of the metal chelator 2,3-dimercapto-1-propanesulfonic acid (DMPS). Can J Physiol Pharmacol 88: 141-146.

Vallon V, Eraly SA, Rao SR, Gerasimova M, Rose M, Nagle M, Anzai N, Smith T, Sharma K, Nigam SK, et al. (2012) A role for the organic anion transporter OAT3 in renal creatinine secretion in mice. Am $J$ Physiol Renal Physiol 302: F1293-F1299.

Varghese B, Barriere H, Carbone CJ, Banerjee A, Swaminathan G, Plotnikov A, Xu P, Peng J, Goffin V, Lukacs GL, et al. (2008) Polyubiquitination of prolactin receptor stimulates its internalization, postinternalization sorting, and degradation via the lysosomal pathway. Mol Cell Biol 28:5275-5287.

Villar SR, Brandoni A, Anzai N, Endou H, and Torres AM (2005) Altered expression of rat renal cortical OAT1 and OAT3 in response to bilateral ureteral obstruction. Kidney Int 68:2704-2713.

Wang C, Wang C, Liu Q, Meng Q, Cang J, Sun H, Peng J, Ma X, Huo X, and Liu K (2014) Aspirin and probenecid inhibit organic anion transporter 3-mediated renal uptake of cilostazol and probenecid induces metabolism of cilostazol in the rat. Drug Metab Dispos 42:996-1007.

Wang H, Xu D, Toh MF, Pao AC, and You G (2016) Serum- and glucocorticoidinducible kinase SGK2 regulates human organic anion transporters 4 via ubiquitin ligase Nedd4-2. Biochem Pharmacol 102:120-129.

Wang H, Zhang J, and You G (2019) Activation of protein kinase A stimulates SUMOylation, expression, and transport activity of organic anion transporter 3. AAPS J 21:30.

Wang L and Sweet DH (2013) Renal organic anion transporters (SLC22 family): expression, regulation, roles in toxicity, and impact on injury and disease. AAPS $J$ 15:53-69.

Ward F, Dunne O, Crotty TB, Fennelly D, Watson A, and Holian J (2012) Successful use of combined high cut-off haemodialysis and bortezomib for acute kidney injury associated with myeloma cast nephropathy. Ir Med J 105:148-149.

Xu D, Wang H, and You G (2016a) Posttranslational regulation of organic anion transporters by ubiquitination: known and novel. Med Res Rev 36:964-979.

Xu D, Wang H, Zhang Q, and You G (2016b) Nedd4-2 but not Nedd4-1 is critical for protein kinase C-regulated ubiquitination, expression, and transport activity of human organic anion transporter 1. Am J Physiol Renal Physiol 310 F821-F831. 
Xu D and You G (2017) Loops and layers of post-translational modifications of drug transporters. Adv Drug Deliv Rev 116:37-44.

Xu D, Zhang J, Zhang Q, Fan Y, Liu C, and You G (2017) PKC/Nedd4-2 signaling pathway regulates the cell surface expression of drug transporter hOAT1. Drug Metab Dispos 45:887-895.

Xue X, Gong LK, Maeda K, Luan Y, Qi XM, Sugiyama Y, and Ren J (2011) Critical role of organic anion transporters 1 and 3 in kidney accumulation and toxicity of aristolochic acid I. Mol Pharm 8:2183-2192.

Yang YP, Hu LF, Zheng HF, Mao CJ, Hu WD, Xiong KP, Wang F, and Liu CF (2013) Application and interpretation of current autophagy inhibitors and activators. Acta Pharmacol Sin 34:625-635.

You G (2002) Structure, function, and regulation of renal organic anion transporters. Med Res Rev 22:602-616.

You G (2004) Towards an understanding of organic anion transporters: structurefunction relationships. Med Res Rev 24:762-774.

Zeng Y, Zhang R, Wu J, Liu M, Peng W, Yu X, and Yang X (2012) Organic anion transporter 1 (OAT1) involved in renal cell transport of aristolochic acid I. Hum Exp Toxicol 31:759-770.

Zeniya M, Mori T, Yui N, Nomura N, Mandai S, Isobe K, Chiga M, Sohara E, Rai T, and Uchida S (2017) The proteasome inhibitor bortezomib attenuates renal fibrosis in mice via the suppression of TGF- $\beta 1$. Sci Rep 7:13086.
Zhang H, Liu Z, Huang L, Hou J, Zhou M, Huang X, Hu W, and Liu Z (2017) The short-term efficacy of bortezomib combined with glucocorticoids for the treatment of refractory lupus nephritis. Lupus 26:952-958.

Zhang Q, Hong M, Duan P, Pan Z, Ma J, and You G (2008) Organic anion transporter OAT1 undergoes constitutive and protein kinase C-regulated trafficking through a dynamin- and clathrin-dependent pathway. J Biol Chem 283:32570-32579.

Zhang Q, Li S, Patterson C, and You G (2013) Lysine 48-linked polyubiquitination of organic anion transporter-1 is essential for its protein kinase C-regulated endocytosis. Mol Pharmacol 83:217-224.

Zhang Q, Suh W, Pan Z, and You G (2012) Short-term and long-term effects of protein kinase $\mathrm{C}$ on the trafficking and stability of human organic anion transporter 3 . Int $J$ Biochem Mol Biol 3:242-249.

Zhao N, Zhang AS, Worthen C, Knutson MD, and Enns CA (2014) An iron-regulated and glycosylation-dependent proteasomal degradation pathway for the plasma membrane metal transporter ZIP14. Proc Natl Acad Sci USA 111:9175-9180.

Address correspondence to: Guofeng You, Department of Pharmaceutics, Rutgers, The State University of New Jersey, 160 Frelinghuysen Rd., Piscataway, NJ 08854. E-mail: gyou@pharmacy.rutgers.edu 